Article

\title{
Consumption of fortified infant cereals and association with Anemia and growth: insights from Demographic Health Surveys (DHS) data analysis
}

\author{
Patrick Detzel1,*, Alberto Prieto-Patron'1 \\ 1 Nestlé Research, 1000 Lausanne 26, Vaud, Switzerland \\ * Correspondence: patrick.detzel@rdls.nestle.com;
}

\begin{abstract}
After approximately 6 months of age, term breastfed infants are increasingly depending on other sources of iron to avoid iron deficiency anemia. The appropriate complementary feeding must include a balance composition of foods containing an adequate amount of macro- and micronutrients to reduce the risk of iron deficiency anemia. This study aims to compare the anemia and growth status of infants receiving commercial fortified infant cereals (FIC) with infants not receiving them. We use all complete multiple Demographic Health Surveys (DHS) from 2005 to 2018 to understand global infant feeding patterns. To better control for the strong household wealth effect in nutritional choices and possibly health awareness, we use propensity score technique as applied in outcome research to better control the effect of covariates. After matching and controlling for confounders, we did find a significant association between reduced risk of anemia and consumption of FIC. After matching and adjusting for confounders the small but positive effects of consumption of FIC on Height for age z-score and Weight for Height Z-score are no longer statistically significant.
\end{abstract}

Keywords: fortified infant cereals; anemia; growth; infants

\section{Introduction}

WHO recommends that infants should be exclusively breastfed for the 6 month of life before the introduction of complementary foods [1]. Breast milk is an ideal food for young infants because of its unique nutrient profile. However, breast milk alone beyond the 6th month of life may lead to insufficiencies of some nutrients such as iron, zinc, vitamin K and vitamin D [2]. After 6 month, term and breastfed infants need additional sources of iron to avoid iron deficiency because of the depletion of iron stores linked to rapid growth and low content of iron in breast milk [3, 4]. Complementary foods are required to meet the macro- and micronutrient requirements[5].

Complementary feeding is defined as providing nutrient-containing foods or liquids along with human milk and do not displace human milk [1]. To meet the gap of breast milk nutritional decrease of macro- and micronutrients, good quality of complementary feeding are needed. This complementary food should include a balance composition of foods containing adequate amount of macro and micronutrients, especially iron, to avoid iron deficiency anemia.

In developing countries, incidence and severity of anemia in infant and young child aged 6 to 23 months is very high [6]. Iron deficiency anemia in children to a large part linked to low intake of iron in complementary foods.

Strategies for achieving sufficient iron intakes include foods rich of bioavailable iron such as meat, the use of fortified complementary foods, or supplements [7]. However as many epidemiological studies have shown the reality of infant feeding in low and middle income countries is characterized by low dietary diversity [8], low nutrient density [9] and increasing use of soft drinks and sugary snacks [10].

Both home-made and commercial complementary foods (fortified infant cereals FIC) are equally acceptable alternatives for infant feeding [11]. Home-made complementary food could provide a broad range of different flavors and textures which affects the development of diet diversity, nutrient 
intakes and quality of infant growth but are low in terms of micronutrient density $[12,13]$. Homemade fortification of complementary feeding with micronutrient supplements has been strongly endorsed by WHO to increase the nutrient density of homemade porridges [14]. Fortified commercial infant cereals provides iron and other micronutrients which are needed by infants and have been associated with decreased risk of iron deficiency anemia [15].

The objective of this analysis is to assess if the consumption of fortified complementary feeding which has been shown in systematic reviews [15-17] and cross-sectional surveys [6, 18] to reduce anemia, is associated with reduced risk of anemia but also with other metabolic markers such as height for age or weight for height. In a small cross sectional survey Irawan et al. observed that infants fed with commercially infant cereals have higher hemoglobin, serum ferritin and serum iron levels than those with home-made complementary food and also had a lower risk of being stunted and wasted [19]. No significant differences were observed in relation with weight-for-age z-scores (underweight). In a longitudinal observational survey in Indonesia, Diana et al. observed that infants fed with FICs had higher intakes of calcium, iron, and vitamins $A$ and $C$ and positive relations existed for 9-month consumption of iron-rich/iron fortified infant foods with length-for-age Z-score (LAZ) at 12 months, and for fortified infant foods alone with both LAZ and weight-for-age Z-score 12 months.

Similarly in the context of randomized control trials, some interventions had additional secondary endpoints next to the anemia status. The effects of fortification on metabolic outcomes was not clear. However, none of the interventions had a control arm with no fortified infant cereals meaning that only the effectiveness of the fortification of commercial cereals or homemade porridges on growth status was assessed and not the fortified cereals versus a group of infant fed with homemade porridges / cereals [15].

In high income countries one longitudinal survey has shown in increase weight gains for infants drinking milk cereals at age 12 months, 18 month [20] and 5 years[21]. This relation has to be set in the context of high-income countries where infants are fed with a large number of food groups and high dietary diversity. According to the authors, the use of milk cereal drink use was associated with low parental education and maternal obesity and were given to reduce troubled sleep which could lead to overfeeding. An additional specificity of the two longitudinal studies is that they looked at cereals in a liquid form (bottled fed) which is not equivalent to a porridge type of semi-solid food consumed (spoon fed).

In developing countries, in contrast, fortified cereals are consumed by higher income groups and are perceived by caretakers as nutritionally superior, convenient food group [22, 23]. Infants receiving FICs had better micronutrients status than infants fed with home made porridges [23]. What raises more concern in this setting with regard to childhood obesity is the increasing consumption of sugary and savory snack foods and soft drinks by young children amongst different income groups[24, 25]. According to DHS the percentage of infants fed with sugary snack foods in Nigeria in 2008 was between 14 and $48 \%$ (lowest and highest wealth groups) but only 1 to $24 \%$ for fortified infant cereals [26]. An additional difficulty in understanding the role of different food groups on health outcomes, is the timing of complementary feeding. Early introduction which is influenced by nutritional knowledge has been a risk factor for morbidities and accelerated weight gain.

Although overweight and obesity is considered predominantly as a problem of high-income countries, it is an emerging issue in developing countries, especially in the cities [27]. Children in wealthier households could be more exposed to packaged foods that are low in nutrient density. However, the contrary effects have been observed in Kenya. Supermarkets in emerging countries which sell predominantly packaged food do not seem to be a driver of childhood obesity in this setting. The positive effects of supermarkets on child nutrition are channeled through improvements in food variety and dietary diversity [28].

We took advantage of the availability of a rich cross-sectional dataset from the Demographic and Health Surveys (DHS) to explore the link between anemia and healthy growth and consumption of fortified infant cereals in low-income countries. In order to deal with the high heterogeneity of the sample, we exploited a statistical technique called propensity score (PS) matching thanks to which we could run the analysis on a more homogeneous sample. In the next section, we further explained 
the data and methods used in our analysis. In section 3, we presented our results while in section 4 we discuss the interpretation. We summarized our conclusions in section 5 .

\section{Materials and Methods}

\subsection{Propensity score matching}

As we deal with observational data, it is likely that treated and untreated observations differ in their observed and unobserved characteristics. From the extensive literature on the effects of infant feeding (bottle vs breast milk) on obesity and overweight, different suggestions to achieve better causal inference have been proposed, amongst them the propensity score methods. Two recent studies from the United States made use of propensity score techniques to deal with confounding in the analysis of the effect of breastfeeding on obesity and body mass index (BMI). The authors used inverse probability of treatment weights (IPTW) [29] and a generalized propensity score approach (GPS) [30] to match the observations on observable baseline characteristics and found small or no effect of breastfeeding on BMI. Grube M. et al. also applied propensity score methods to reduce the effect of confounding factors, but with a difference: the authors did not include the propensity score weights in the regression, but used propensity score matching as a way to select subsamples of breastfed and non-breastfed subjects that were balanced with respect to observed covariates [31]. The authors then used the obtained balanced sample in an adjusted multivariable logistic regression. This means that they had to take into consideration two non-overlapping sets of covariates: one for the calculation of the propensity scores and one to adjust the multivariate regression. Clearly, in order to proceed in this way, one must believe in the assumption of "ignorability", that is, absence of unobserved confounding [31].

One of the advantages of the use of propensity score methods lies in the fact that it allows to identify treated and untreated whose characteristics make them unique to one group or the other, so that, in order to have more comparable observations ("common support"), the mismatched ones can simply be excluded from the sample [31]. Propensity score matching addresses the possible occurrence of selection bias and reverse causality and to estimate treatment effects when treatment is endogenous to the outcome. For example, infants born in wealthier households would have a higher probability of being overweight and been feed with fortified cereals. Matching mimics a randomized experiment i.e. conditional on a set of observables there is some unspecified mechanism that randomizes people into treatment. Given the high heterogeneity of our sample, we exploited a propensity score technique to match observations according to a set of confounding covariates and obtain a more homogeneous sample on which we than run standard regressions.

To balance the groups of babies fed with FIC and not fed with FIC and to estimate the average treatment effect for the outcome variables we used Stata command nnmatch [32]. With this command we applied the nearest neighbor matching technique across the variables age of infants in months, first child born, low birth weight, wanted pregnancy, mother currently working, maternal, Educational level with an exact match on survey, wealth quintile and country sub-national region.

\subsection{Data flow}

To generate the data sets suitable to run propensity score, we first selected all infants from the DHS surveys from 2005 to 2018.122 surveys were used and 271'478 children aged between 12 and 24 months were extracted from the surveys. Error! Reference source not found. shows the flow chart of the inclusion/exclusion of young children included in the analysis for hemoglobin. All step until level 5 are linked to missing information in the data sets. Not all surveys collected routinely the complete set of information of young children. Two flow charts were used. One for generating two groups to analyze hemoglobin, a second for anthropometric outcomes.

$148^{\prime} 394$ observations were dropped between level 1 and 2 due to missing/not collected information on hemoglobin. 7'934 observations were dropped between level 2 and 3 due to missing information on food groups. 772 observations were dropped between level 3 and 4 due to missing information for the variables used in the matching equation. 40'620 observations were dropped 
between level 4 and 5 due to missing information on covariables used in the regressions. Finally, 31'799 observations were dropped because they were not matched to the FICs group.

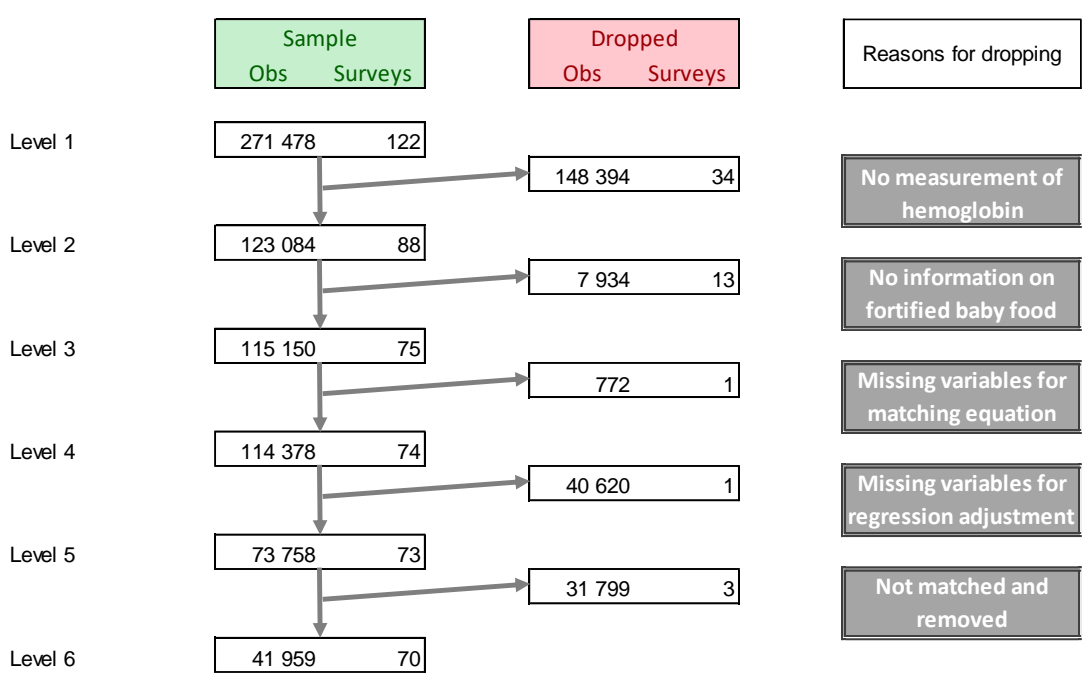

Figure 1: Flow chart on young children included in the analysis of hemoglobin

The last level is a central one as it helps generating two 'synthetic' groups which are more comparable and reduces the collinearity between the variables: wealthier households buy more fortified IC but are also more prone to have a higher health literacy that would influence dietary choices but also impact the health status of the infant.

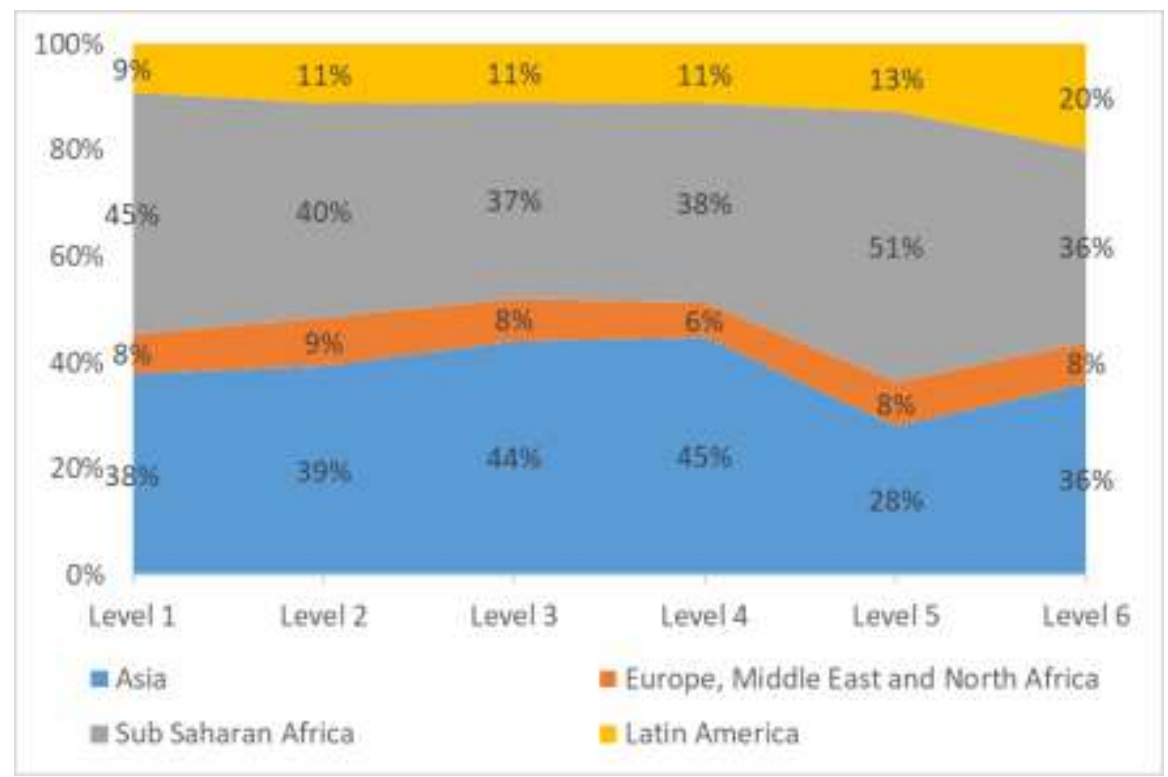

Figure 2: Sample regional representation at different levels of the analysis

Error! Reference source not found. illustrates how the different selection steps influence the composition of the data set. The main change is the relative number of young children in sub Saharan Africa especially at level 5. This is due to the fact that the percentage of children fed with commercial cereals are much lower than other parts of the developing world which increases the heterogeneity of this group and decreasing the power of matching.

\section{Results}

\subsection{Descriptive}


As illustrated in Table 1, the two groups (infants fed with FIC and not) differ considerably in terms of socio-economics dimensions of the mothers and the household. Children fed with FIC are born of mothers of higher education status, of higher BMI and are raised in wealthier urban households. After the process of the propensity matching, these differences have been reduced substantially but still statistically significant. A more stringent matching approach (one to one) would have led to a substantial drop out of observations in both matched groups. Here we used a one-to-n matching. However, the heterogeneity was substantially reduced, and further statistical regressions would further control the confounding effects.

Table 1: Characteristics of the young children according to consumption of fortified infant cereals (matched sample)

\begin{tabular}{|c|c|c|c|c|c|}
\hline & \multicolumn{2}{|c|}{ Consumer } & \multicolumn{2}{|c|}{ No Consumer } & \multirow[t]{2}{*}{$p$-value } \\
\hline & mean $/ \%$ & $S D$ & mean & $S D$ & \\
\hline Child age in months & 17,03 & 3,4 & 17,24 & 3,4 & 0.0000 \\
\hline First born child & $36,00 \%$ & & $26,60 \%$ & & 0.0000 \\
\hline Low birth weight & $20,20 \%$ & & $23,00 \%$ & & 0.0000 \\
\hline Born by C-section & $19,90 \%$ & & $11,10 \%$ & & 0.0000 \\
\hline Wanted pregnancy & $74,60 \%$ & & $75,80 \%$ & & 0.0000 \\
\hline Mother currently working & $36,10 \%$ & & $37,20 \%$ & & 0.4204 \\
\hline Maternal BMI & & & & & 0.0000 \\
\hline lower than 18.5 & $12,70 \%$ & & $18,10 \%$ & & \\
\hline Between 18.5 and 25 & $57,70 \%$ & & $60,40 \%$ & & \\
\hline Higher than 25 & $29,60 \%$ & & $21,50 \%$ & & \\
\hline Mothers education & & & & & 0.0000 \\
\hline No education & $17,30 \%$ & & $33,10 \%$ & & \\
\hline Primary education & $26,70 \%$ & & $26,20 \%$ & & \\
\hline Secondary education and above & $56,10 \%$ & & $40,70 \%$ & & \\
\hline Wealth quintile & & & & & 0.0000 \\
\hline Lowest (reference) & $15,00 \%$ & & $25,70 \%$ & & \\
\hline Lower & $16,10 \%$ & & $21,90 \%$ & & \\
\hline Middle & $18,60 \%$ & & $18,90 \%$ & & \\
\hline Higher & $21,10 \%$ & & $16,80 \%$ & & \\
\hline Highest & $29,20 \%$ & & $16,60 \%$ & & \\
\hline Urban (vs rural) & $45,20 \%$ & & $30,40 \%$ & & 0.0000 \\
\hline Dietary Diversity Score $>4$ & $47,90 \%$ & & $24,30 \%$ & & 0.0000 \\
\hline Observations & 9280 & $20.9 \%$ & 35217 & $79.1 \%$ & \\
\hline
\end{tabular}

\subsection{Results association FIC and anemia}




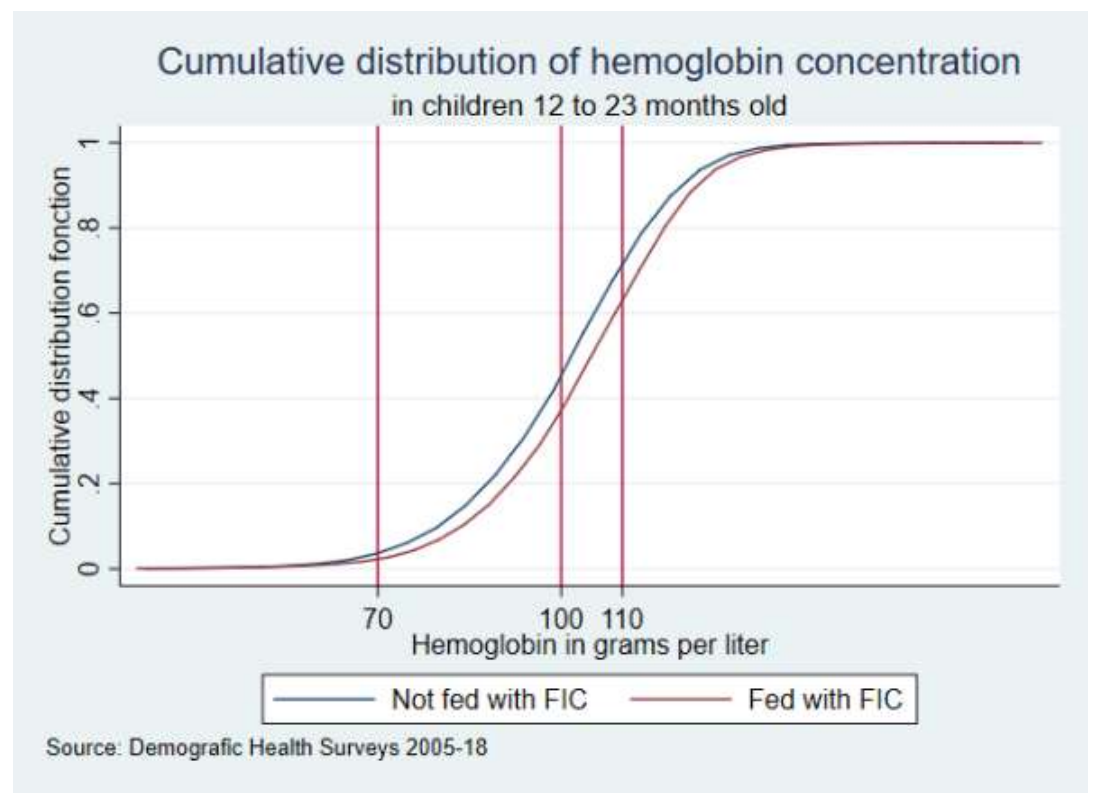

Figure 3: Cumulative distribution on hemoglobin on the matched sample

The cumulative distribution of the hemoglobin status after matching point at a substantial shift of the cumulative distribution from the not FIC fed babies to the FIC fed infants. $62 \%$ of the infants fed with FIC are anemia (less than $110 \mathrm{~g} / \mathrm{L}$ : mild to severe) and $72 \%$ for the non FIC fed infants. $38 \%$ of the infants fed with FIC are below the threshold on moderate anemia $(100 \mathrm{~g} / \mathrm{L})$, were as $45 \%$ of the infants not receiving FIC are moderate to severe deficient. To assess if these two cumulative distribution curves are statistically different, we will run additional regressions on the matched samples.

In the matched but unadjusted analysis (before regressions) Table 2 being fed with FIC increase hemoglobin by $1.63 \mathrm{~g} / \mathrm{L}$ or is expressed in an logistic regression reduced the Odd ration by $21 \%$. The effect of the propensity score in reducing the heterogeneity between the two groups can be seen in the reduction of the "treatment" effect of being fed with FIC from 1,86g/1 to 1,63. After controlling for covariables and using fix-effects of the survey years and subnational regions, this effect is roughly halved $(0.8 \mathrm{~g} / \mathrm{L}$ and RR of .11) but still highly statistically significant. Controlling for covariables on matched groups indicates that infants fed with FICs have a significant improve hemoglobin status $(0.8 \mathrm{~g} / \mathrm{L})$ or a reduced probability of being anemic. This is very similar to the estimated effect estimated by Prieto et al. although they used another statistical technique[6].

Table 2: association between fortified infant cereals consumption and hemoglobin $(\mathrm{Hb} / \mathrm{l}<110)$

\begin{tabular}{|c|c|c|c|c|c|c|c|c|}
\hline & \multicolumn{2}{|c|}{$\begin{array}{l}\text { Unmatched sample, } \\
\text { unadjusted analyses }\end{array}$} & \multicolumn{2}{|c|}{$\begin{array}{l}\text { Unmatched sample, } \\
\text { adjusted analyses }\end{array}$} & \multicolumn{2}{|c|}{$\begin{array}{c}\text { Matched sample, } \\
\text { unadjusted analyses }\end{array}$} & \multicolumn{2}{|c|}{$\begin{array}{l}\text { Matched sample, } \\
\text { adjusted analyses }\end{array}$} \\
\hline & $\begin{array}{c}\text { Linear } \\
\text { reg }\end{array}$ & $\begin{array}{l}\text { Logistic } \\
\text { reg }\end{array}$ & $\begin{array}{c}\text { Linear } \\
\text { reg }\end{array}$ & $\begin{array}{c}\text { Logistic } \\
\text { reg }\end{array}$ & $\begin{array}{c}\text { Linear } \\
\text { reg }\end{array}$ & $\begin{array}{l}\text { Logistic } \\
\text { reg }\end{array}$ & $\begin{array}{c}\text { Linear } \\
\text { reg }\end{array}$ & $\begin{array}{c}\text { Logistic } \\
\text { reg }\end{array}$ \\
\hline Fortified baby food & $1,86^{*}$ & $0,78^{*}$ & $1,12^{*}$ & $0,85^{*}$ & $1,63^{*}$ & $0,79^{*}$ & $0,97^{*}$ & $0,86^{*}$ \\
\hline Child age in months & & & $0,34^{*}$ & $0,94^{*}$ & & & $0,33^{*}$ & $0,94^{*}$ \\
\hline First born child & & & 0,03 & 0,97 & & & 0,25 & 0,96 \\
\hline Low birth weight & & & $-1,46^{*}$ & $1,16^{*}$ & & & $-1,49^{*}$ & $1,17^{*}$ \\
\hline Born by C-section & & & 0,12 & 0,93 & & & 0,17 & 0,9 \\
\hline Wanted pregnancy & & & $0,34^{*}$ & $0,95^{*}$ & & & $0,44^{*}$ & 0,96 \\
\hline $\begin{array}{l}\text { Mother currently } \\
\text { working }\end{array}$ & & & $0,30^{*}$ & 0,98 & & & 0,2 & 1 \\
\hline
\end{tabular}

Maternal BMI 


\begin{tabular}{|c|c|c|c|c|}
\hline Between 18.5 and 25 & $0,92^{*}$ & $0,88^{*}$ & $1,15^{*}$ & $0,83^{*}$ \\
\hline Higher than 25 & $1,70^{*}$ & $0,84^{*}$ & $1,92^{*}$ & $0,79^{*}$ \\
\hline \multicolumn{5}{|l|}{ Mothers education } \\
\hline \multicolumn{5}{|l|}{ No education (reference) } \\
\hline $\begin{array}{l}\text { Primary education (1 to } \\
\text { 5yrs) }\end{array}$ & $0,69^{*}$ & $0,91^{*}$ & $0,58^{*}$ & 0,93 \\
\hline $\begin{array}{l}\text { Secondary education and } \\
\text { above }\end{array}$ & $1,62^{*}$ & $0,80^{*}$ & $1,46^{*}$ & $0,83^{*}$ \\
\hline \multicolumn{5}{|l|}{ Wealth quintile } \\
\hline \multicolumn{5}{|l|}{ Lowest (reference) } \\
\hline Lower & $0,40^{*}$ & $0,93^{*}$ & 0,32 & 0,93 \\
\hline Middle & $0,85^{*}$ & $0,89^{*}$ & $0,75^{*}$ & $0,87^{*}$ \\
\hline Higher & $1,72^{*}$ & $0,83^{*}$ & $2,01^{*}$ & 0,78 \\
\hline Highest & $3,82^{*}$ & $0,64^{*}$ & $4,14^{\prime \prime}$ & 0,6 \\
\hline Urban (vs rural) & $-0,23$ & 1,04 & $-0,47^{*}$ & $1,08^{*}$ \\
\hline Dietary Diversity Score $>4$ & 0,14 & 0,98 & 0,2 & 0,98 \\
\hline
\end{tabular}

From the covariates used in the regressions, the most significant ones are linked to the wealth status of the household in which the infant was born, the educational status of the mother but also the weight of the mother. Having a mother of a BMI under 18.5 is associated with increased risk for the child to be anemic. This could be explained by the association of BMI and maternal anemia which is a risk factor for infant anemia[33].

Low birth weight is associated with a significantly higher risk of being anemic between 12 and 18 months. This effect has been shown in other epidemiological studies[34].

Dietary diversity is not significantly associated with hematological status as also shown in Ghana[35]. Being fed with different food groups after controlling for covariates in not associated with the anemia status.

\subsection{Association consumption of FIC and stunting}

Current knowledge on association of infant feeding and food groups and growth focuses on breastfeeding and dietary diversity score and stunting. Global and local epidemiological studies have shown the important role of dietary diversity on the anthropometric status of infants and young children[36-38]. Stunting is been defined as Height for age z score under -2. Here we will focus on the specific effect of FIC. Although some clinical trials have shown some marginal effects on reducing stunting[39], we would not expect a significant effect of FIC compared to non FIC on stunting. 


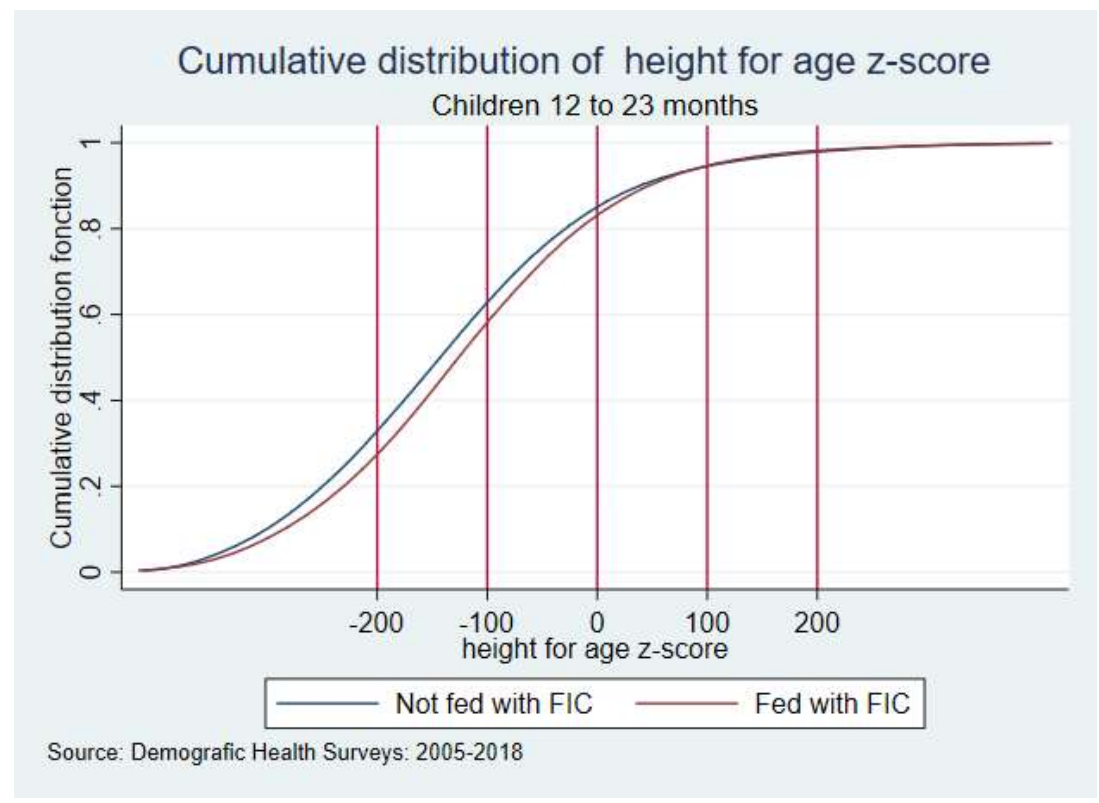

Figure 4:Cumulative distribution on Heigh-for-age z-score on the matched samples

Both cumulative distribution are close to each other which indicates small negligible differences between the two groups. After matching $30 \%$ of the infants of the DHS are stunted (under -200 which stands for -2Z) of infants not fed with FICs are stunted. This rate is slightly lower than the estimates of Argaw et al. but can be explained by the large weight of India in their study[40] but very similar to the one estimated by De Onis[41]. In the FICs group this percentage is around $28 \%$.

Table 3: association between fortified infant cereals consumption and Height-for-age score (HAZ<-2)

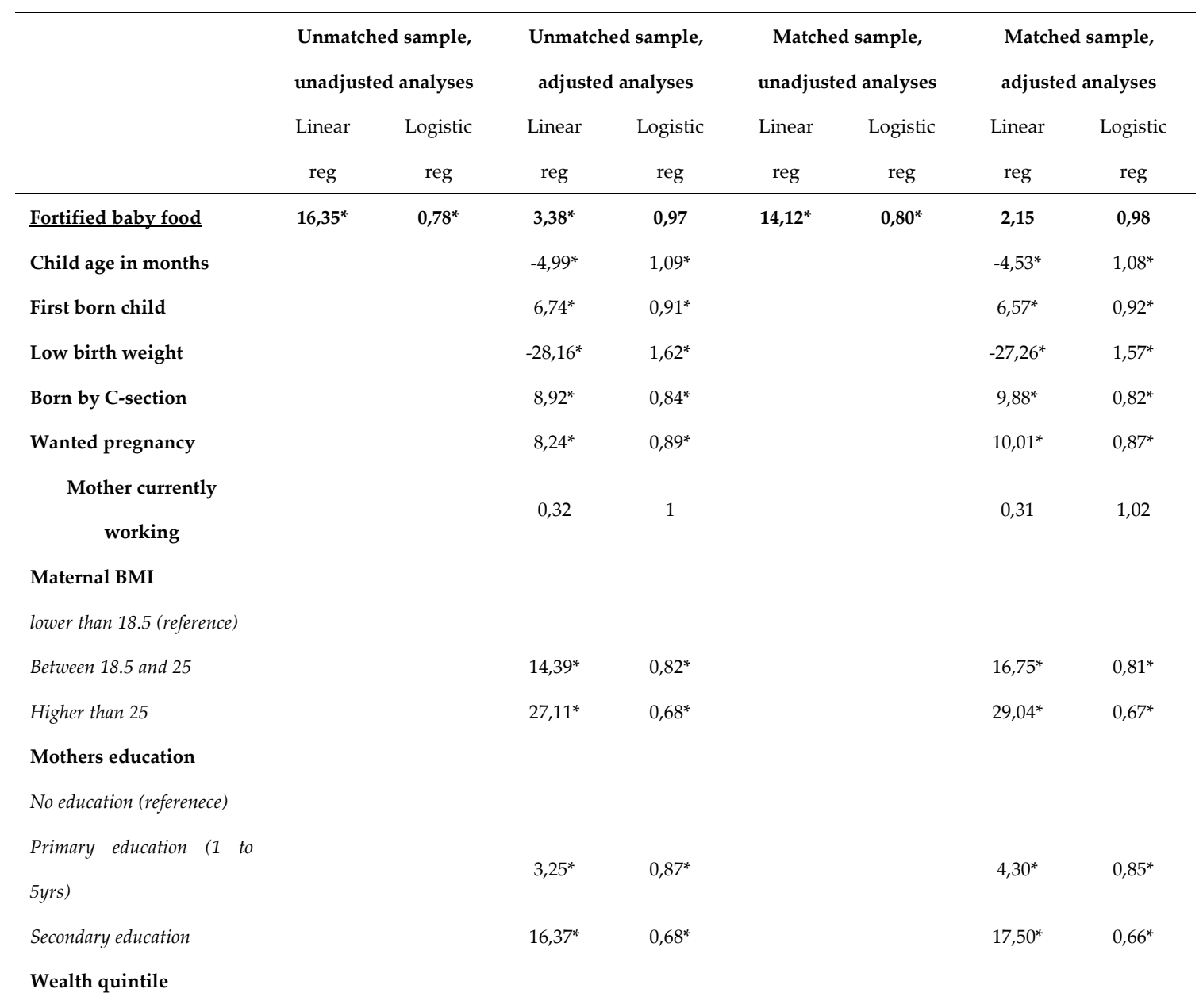




$\begin{array}{lcccc}\text { Lowest (reference) } & & & & \\ \text { Lower } & 7,36^{*} & 0,85^{*} & 10,19^{*} & 0,83^{*} \\ \text { Middle } & 14,65^{*} & 0,75^{*} & 18,61^{*} & 0,72^{*} \\ \text { Higher } & 20,79^{*} & 0,64^{*} & 26,98^{*} & 0,59^{*} \\ \text { Highest } & 42,58^{*} & 0,46^{*} & 49,05^{*} & 0,43^{*} \\ \text { Urban (vs rural) } & 2,47^{*} & 1,02 & 3,15^{*} & 1,03 \\ \text { Dietary Diversity Score>4 } & 3,10^{*} & 0,94^{*} & 2,52^{*} & 0,94^{*}\end{array}$

Infants fed with FICs seems to be less stunted in the unadjusted estimations than not FICs but when matched and adjusted regressions are used the effects is not significant anymore. Being fed with FICs is not statistically significantly different from the control groups when it comes to reducing the risk of stunting.

Maternal education and household wealth having a much larger effects on stunting. Maternal education especially if secondary education and above have a significant effect on stunting. Low maternal weight as well as low birth weights are also important risk factors for stunting. These are well known risks factors for infant stunting [42-44]. To a lesser degree nutritional diversity is also associated with a lower risk of stunting[38].

\subsection{Association of FIC on Weight-for-Height Z-score}

Weight for height score are indicators of wasting if z-score below -2 and of overweight is z-score above 2. In the matched samples both extremes of weight score values are relatively small fractions of the infant populations, especially for overweight. That is why we have used a lower threshold value of $z$-score above 1 in the logistic regressions which is describing infant at risk of being overweight or being overweight.

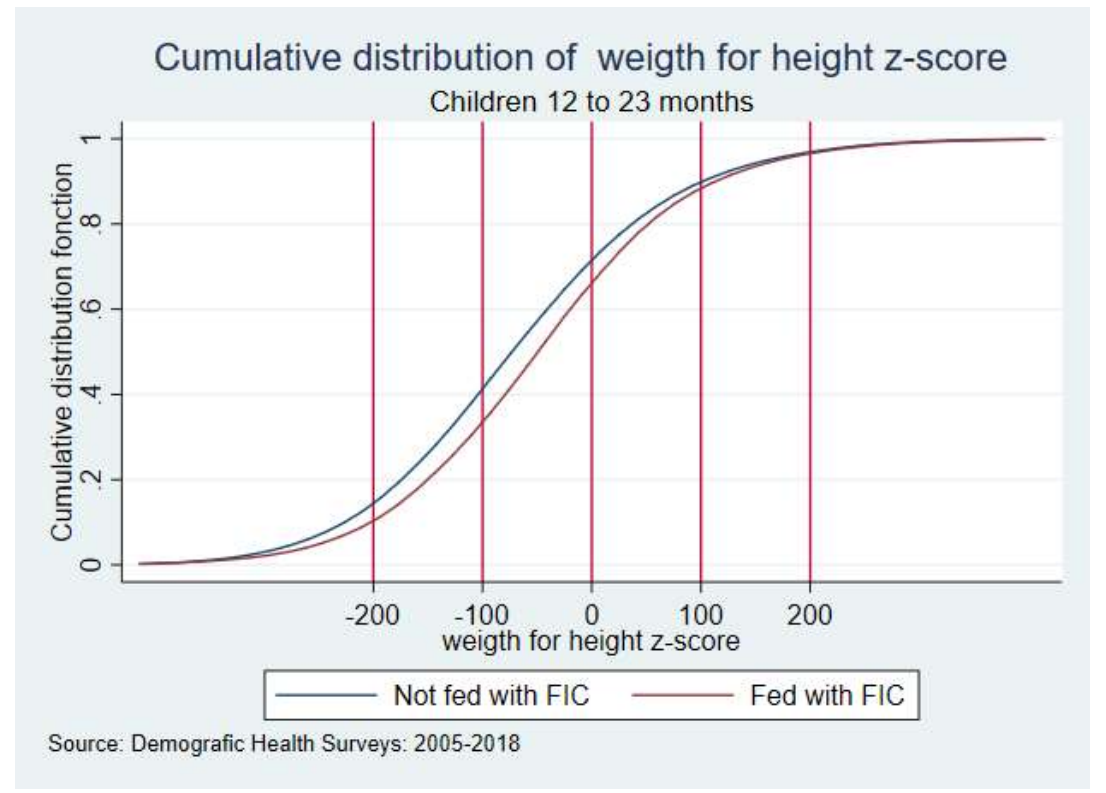

Figure 5:Cumulative distribution on Weight-for-Height z-score on the matched samples

$11 \%$ of the infants fed with FICs are wasted, $16 \%$ of infants not receiving FIC are wasted. This is in line with global estimates[45]. $11 \%$ of the infants of both groups are above $z>1$ which is the cutoff point for infants are risk of being overweight. This is in line with the estimated global prevalence[46].

In Table 4 the small effect of FIC on the WHZ score is not statistically significant once we control for confounding effects. The small odd ratio in the matched but unadjusted effect of $9 \%$ of being at risk of overweight is not significant once we adjust for socio-economic and maternal confounders. 
The characteristics having the largest effects on the BMI of infants are the wealth status of the household and maternal weight although these factors are having only a very marginal effects.

Table 4 Association between fortified infant cereals consumption and Weight-for-height

\begin{tabular}{|c|c|c|c|c|c|c|c|c|}
\hline & \multicolumn{2}{|c|}{$\begin{array}{l}\text { Unmatched sample, } \\
\text { unadjusted analyses }\end{array}$} & \multicolumn{2}{|c|}{$\begin{array}{l}\text { Unmatched sample, } \\
\text { adjusted analyses }\end{array}$} & \multicolumn{2}{|c|}{$\begin{array}{c}\text { Matched sample, } \\
\text { unadjusted analyses }\end{array}$} & \multicolumn{2}{|c|}{$\begin{array}{r}\text { Matched sample, } \\
\text { adjusted analyses }\end{array}$} \\
\hline & $\begin{array}{l}\text { Linear } \\
\text { reg }\end{array}$ & $\begin{array}{l}\text { Logistic } \\
\text { reg }\end{array}$ & $\begin{array}{l}\text { Linear } \\
\text { reg }\end{array}$ & $\begin{array}{l}\text { Logistic } \\
\text { reg }\end{array}$ & $\begin{array}{l}\text { Linear } \\
\text { reg }\end{array}$ & $\begin{array}{l}\text { Logistic } \\
\text { reg }\end{array}$ & $\begin{array}{l}\text { Linear } \\
\text { reg }\end{array}$ & $\begin{array}{l}\text { Logistic } \\
\text { reg }\end{array}$ \\
\hline Fortified baby food & $6,19^{*}$ & $1,09^{*}$ & $-0,81$ & 0,98 & $6,47^{*}$ & $1,09^{*}$ & 0,17 & 1 \\
\hline Child age in months & & & $-0,34^{*}$ & 0,99 & & & $-0,37^{*}$ & 0,99 \\
\hline First born child & & & $5,85^{*}$ & $1,18^{*}$ & & & $6,65^{*}$ & 1,19 \\
\hline Low birth weight & & & $-25,16^{*}$ & $0,71^{*}$ & & & $-24,17^{*}$ & 0,74 \\
\hline Born by C-section & & & $8,77^{*}$ & $1,21^{*}$ & & & $7,49^{*}$ & 1,22 \\
\hline Wanted pregnancy & & & $2,97^{*}$ & $1,13^{*}$ & & & $2,64^{*}$ & 1,15 \\
\hline $\begin{array}{l}\text { Mother currently } \\
\text { working }\end{array}$ & & & $-0,08$ & 0,97 & & & $-0,29$ & 0,96 \\
\hline Maternal BMI & & & & & & & & \\
\hline lower than 18.5 (reference) & & & & & & & & \\
\hline Between 18.5 and 25 & & & $29,34^{*}$ & $1,60^{*}$ & & & $27,27^{*}$ & $1,56^{*}$ \\
\hline Higher than 25 & & & $52,72^{*}$ & $2,44^{*}$ & & & $50,76^{*}$ & $2,34^{*}$ \\
\hline Mothers education & & & & & & & & \\
\hline No education (reference) & & & & & & & & \\
\hline $\begin{array}{l}\text { Primary education (1 to } \\
\text { 5yrs) }\end{array}$ & & & $4,89^{*}$ & 1,06 & & & $4,87^{*}$ & 1 \\
\hline Secondary education & & & $11,56^{*}$ & $1,20^{*}$ & & & $12,27^{*}$ & $1,18^{*}$ \\
\hline Wealth quintile & & & & & & & & \\
\hline Lowest (reference) & & & & & & & & \\
\hline Lower & & & 2,03 & 1 & & & 2,02 & 0,99 \\
\hline Middle & & & $6,16^{*}$ & $1,08^{*}$ & & & $7,66^{*}$ & 1,04 \\
\hline Higher & & & $8,36^{*}$ & 1,06 & & & $9,74^{*}$ & 1,05 \\
\hline Highest & & & $17,53^{*}$ & $1,23^{*}$ & & & $18,96^{*}$ & $1,18^{*}$ \\
\hline Urban (vs rural) & & & $-0,91$ & 1 & & & $-0,46$ & 1,04 \\
\hline Dietary Diversity Score >4 & & & 1,1 & 1 & & & $1,12^{*}$ & 1 \\
\hline
\end{tabular}

\section{Discussion}

Improper complementary feeding practices are a common problem in developing countries. A recent cross-sectional study was performed in 392 Indonesian infants aged 6-23 months with the goal of analyzing complementary feeding practices and nutritional status[47]. Results from this study indicated that complementary feeding practices were sub-optimal, particularly with respect to minimal dietary diversity, hygiene practices, and the consumption of foods rich in iron and vitamin A. Based on the Indonesia Individual Food Consumption Survey (IFCS) 2014, Egayanti et al. confirmed that the intake of fat, protein, iron, zinc and calcium among infants in that middle income country was inadequate and estimated that this was largely due to nutrient poor home-made porridges. Iron, zinc and calcium intake of Indonesian infants were mainly from fortified infant cereals, but the amount of FIC consumption was very small compared to homemade porridges[48]. 
In its Global Strategy for Infant and Young Child feeding, the WHO/UNICEF recommend the use of affordable and locally available foods[1]. In an Indonesian study assessing the effectiveness of complementary feeding recommendations (CFRs) in improving maternal knowledge, feeding practices, and child intake of key problem nutrients including iron, Fahmida et al. found that a major limiting factor was economic access to nutrient-dense foods[12]. Typical of many rural Indonesian families, poverty and household food insecurity were prevalent in the population of this study. This suggests that cost is an important factor that shapes child feeding practices, particularly among lower-income populations.

Taken together, these findings suggest that for iron and other key micronutrients, complementary feeding regimens based solely upon local foods may not be sufficient to ensure dietary adequacy, underscoring the need for alternative strategies[12].

The fortification of widely-used complementary foods provides a means to circumvent these problems[16]. Improvements in manufacturing techniques and the local production of fortified blended cereal products have lowered costs and made these foods more affordable for low-income families[49]. Compared to other strategies such as micronutrient supplementation or home based fortification, the use of food-based strategies has been shown to be more sustainable over the long run[23]. The advantages of fortified complementary foods such as infant cereals include the ability to provide an appropriate balance of nutrients, the potential for minimizing microbial contamination compared to home-prepared foods, and the time savings for caregivers[49]. Furthermore, the longer shelf-life of these foods would render these a practical alternative for rural families who live far from markets, and who are unable to obtain fresh nutrient-dense foods on a regular basis[23]. The Global Alliance for Improved Nutrition (GAIN) identifies fortified infant cereals as an important vehicle for combating micronutrient deficiencies in infants and young children[50].

Compared to infants not fed with FIC, infants receiving FIC -when controlling for infant specific covariates and household settings- do not have a significant impact on stunting, wasting or overweight. Contrary to what was observed in one high income country with a liquid form, infant cereals are not fed to infants on top of other food groups which could lead to overfeeding. Furthermore we did not observe a reduction of the Diet Diversity in the group of infants fed with FICs as in a small prospective study in Indonesia[18]. The contrary was observed. Close to $50 \%$ of infants fed with FICs had a DDI score above 4 whereas the control group this share was half $(24.3 \%)$ Table 1.

The main weakness of this analysis is the cross-sectional nature of the surveys used. The underlying assumption is that the food intake as collected at the time of the interviews are stable overtime and representative of the feeding groups fed to infants in the weaning period 6-24 months. Although we know that the period of 6 to 12 months is characterized by incremental introduction of weaning food groups, this transition is stable after the 12months. Because we don't know how long and how much an infant has been fed with FIC, we will by construction expect a smaller effect on hemoglobin compared with controlled intervention trials. So the treatment effect would be an underestimate of the consumption of FICs.

\section{Conclusions}

The findings from this study suggest that the consumption of fortified infant cereals is a valuable tool in the arsenal of strategies for reducing the burden of IDA in infants aged 12-23 months. We do not see anthropometric significant effects like reducing the risk of stunting, wasting or increasing the risk of overweight when compared to infants who did not receive fortified infant cereals.

Author Contributions: Patrick Detzel and Alberto Prieto-Patron have contributed to the conceptual design, model building, data analyses and manuscript writing.

Conflicts of Interest: Patrick Detzel and Alberto Prieto-Patron are employees of Nestlé Research

\section{References}


1. WHO, UNICEF., and UNAIDS, Global strategy for infant and young child feeding. 2003: World Health Organization.

2. Mok, E., et al., Diet diversity, growth and adiposity in healthy breastfed infants fed homemade complementary foods. International journal of obesity, 2017. 41(5): p. 776-782.

3. Dewey, K., Guiding principles for complementary feeding of the breastfed child. 2003.

4. Pérez-Escamilla, R., S. Segura-Pérez, and M. Lott, on behalf of the RWJF HER Expert Panel on Best Practices for Promoting Healthy Nutrition, Feeding Patterns, and Weight Status for Infants and Toddlers from Birth to 24 Months. Feeding guidelines for infants and young toddlers: A responsive parenting approach. Durham, NC: Healthy Eating Research, 2017. Feeding Guidelines for Infants and Young Toddlers: A Responsive Parenting Approach. Guidelines for Health Professionals, 2017: p. 118-32.

5. Black, R.E., et al., Maternal and child undernutrition and overweight in low-income and middleincome countries. The lancet, 2013. 382(9890): p. 427-451.

6. Prieto-Patron, A., et al., Association between Anaemia in Children 6 to 23 Months Old and Child, Mother, Household and Feeding Indicators. Nutrients, 2018. 10(9): p. 1269.

7. Organization, W.H., Complementary feeding: family foods for breastfed children. 2000, World Health Organization.

8. Lutter, C.K., et al., Undernutrition, poor feeding practices, and low coverage of key nutrition interventions. Pediatrics, 2011. 128(6): p. e1418-e1427.

9. Lutter, C.K. and R. Lutter, Fetal and early childhood undernutrition, mortality, and lifelong health. Science, 2012. 337(6101): p. 1495-1499.

10. Huffman, S.L., et al., Babies, soft drinks and snacks: a concern in low-and middle-income countries? Maternal \& child nutrition, 2014. 10(4): p. 562-574.

11. Kleinman, R.E., Pediatric nutrition handbook. Actividad Dietética, 2009. 13(1): p. 46.

12. Fahmida, U., Use of fortified foods for Indonesian infants, in Handbook of Food Fortification and Health. 2013, Springer. p. 383-393.

13. Huh, S.Y., et al., Timing of solid food introduction and risk of obesity in preschool-aged children. Pediatrics, 2011. 127(3): p. e544.

14. Guideline, W., use of multiple micronutrient powders for point-of-use fortification of foods consumed by infants and young children aged 6-23 months and children aged 2-12 years. World Health Organization: Geneva, Switzerland, 2016.

15. Eichler, K., et al., Effects of micronutrient fortified milk and cereal food for infants and children: a systematic review. BMC public health, 2012. 12(1): p. 506.

16. Dewey, K.G. and S. Adu-Afarwuah, Systematic review of the efficacy and effectiveness of complementary feeding interventions in developing countries. Maternal \& child nutrition, 2008. 4: p. 2485.

17. Tam, E., et al., Micronutrient Supplementation and Fortification Interventions on Health and Development Outcomes among Children Under-Five in Low-and Middle-Income Countries: A Systematic Review and Meta-Analysis. Nutrients, 2020. 12(2): p. 289.

18. Diana, A., et al., Consumption of fortified infant foods reduces dietary diversity but has a positive effect on subsequent growth in infants from Sumedang district, Indonesia. PLoS One, 2017. 12(4): p. e0175952.

19. Irawan, R., N.A. Widjaja, and M.H. Hanindita, Effect of Different Complementary Feeding on Iron Deficiency Anemia and Growth in Breastfed Infants: Home-Made VS Commercial. Folia Medica Indonesiana, 2019. 55(2): p. 112-116.

20. Almquist-Tangen, G., et al., Milk cereal drink increases BMI risk at 12 and 18 months, but formula does not. Acta paediatrica, 2013. 102(12): p. 1174-1179.

21. Almquist-Tangen, G., et al., Consuming milk cereal drinks at one year of age was associated with a twofold risk of being overweight at the age of five. Acta Paediatrica, 2018.

22. Pelto, G.H. and M. Armar-Klemesu, Balancing nurturance, cost and time: complementary feeding in Accra, Ghana. Maternal \& child nutrition, 2011. 7: p. 66-81.

23. Fahmida, U. and O. Santika, Development of complementary feeding recommendations for 12-23month-old children from low and middle socio-economic status in West Java, Indonesia: contribution of fortified foods towards meeting the nutrient requirement. British Journal of Nutrition, 2016. 116(S1): p. S8S15. 
24. Nordhagen, S., A.M. Pries, and R. Dissieka, Commercial Snack Food and Beverage Consumption Prevalence among Children 6-59 Months in West Africa. Nutrients, 2019. 11(11): p. 2715.

25. Kalanda, B.F., F.H. Verhoeff, and B. Brabin, Breast and complementary feeding practices in relation to morbidity and growth in Malawian infants. European journal of clinical nutrition, 2006. 60(3): p. 401407.

26. Pries, A.M., et al., Consumption of commercially produced snack foods and sugar-sweetened beverages during the complementary feeding period in four African and Asian urban contexts. Maternal \& Child Nutrition, 2017. 13: p. e12412.

27. WHO. Obesity and Overweight. Fact Sheet. Geneva: WHO. 2018 [cited 2019 16.04.2019]; Available from: https://www.who.int/en/news-room/fact-sheets/detail/obesity-and-overweight.

28. Debela, B.L., et al., Supermarket food purchases and child nutrition in Kenya. Global Food Security, 2020. 25: p. 100341.

29. Jenkins, J.M. and E.M. Foster, The effects of breastfeeding exclusivity on early childhood outcomes. American journal of public health, 2014. 104(S1): p. S128-S135.

30. Ma, J.-Q., et al., Association between feeding practices and weight status in young children. BMC pediatrics, 2015. 15(1): p. 97.

31. Grube, M.M., et al., Does breastfeeding help to reduce the risk of childhood overweight and obesity? A propensity score analysis of data from the KiGGS study. PloS one, 2015. 10(3): p. e0122534.

32. Abadie, A., et al., NNMATCH: Stata Module to Compute Nearest-Neighbor Bias-Corrected Estimators. Statistical Software Components. 2004.

33. Mocking, M., et al., Does body mass index early in pregnancy influence the risk of maternal anaemia? An observational study in Indonesian and Ghanaian women. BMC Public Health, 2018. 18(1): p. 873.

34. Kejo, D., et al., Prevalence and predictors of anemia among children under 5 years of age in Arusha District, Tanzania. Pediatric Health Med Ther, 2018. 9: p. 9-15.

35. Saaka, M. and S.Z. Galaa, How is dietary diversity related to haematological status of preschool children in Ghana? Food Nutr Res, 2017. 61(1): p. 1333389.

36. Krasevec, J., et al., Diet quality and risk of stunting among infants and young children in low- and middle-income countries. Matern Child Nutr, 2017. 13 Suppl 2.

37. Marriott, B.P., et al., World Health Organization (WHO) infant and young child feeding indicators: associations with growth measures in 14 low-income countries. Matern Child Nutr, 2012. 8(3): p. 354-70.

38. Perkins, J.M., R. Jayatissa, and S.V. Subramanian, Dietary diversity and anthropometric status and failure among infants and young children in Sri Lanka. Nutrition, 2018. 55-56: p. 76-83.

39. Ekoe, T., et al., Efficacy of an iron-fortified infant cereal to reduce the risk of iron deficiency anemia in young children in East Cameroon. Food Sci Nutr, 2020. 8(7): p. 3566-3577.

40. Argaw, A., et al., Drivers of under-five stunting trend in 14 low-and middle-income countries since the turn of the millennium: a multilevel pooled analysis of 50 Demographic and Health Surveys. Nutrients, 2019. 11(10): p. 2485.

41. De Onis, M. and F. Branca, Childhood stunting: a global perspective. Maternal \& child nutrition, 2016. 12: p. 12-26.

42. Danaei, G., et al., Risk Factors for Childhood Stunting in 137 Developing Countries: A Comparative Risk Assessment Analysis at Global, Regional, and Country Levels. PLoS Med, 2016. 13(11): p. e1002164.

43. Islam, M.M., et al., Risk factors of stunting among children living in an urban slum of Bangladesh: findings of a prospective cohort study. BMC Public Health, 2018. 18(1): p. 197.

44. Vilcins, D., P.D. Sly, and P. Jagals, Environmental Risk Factors Associated with Child Stunting: A Systematic Review of the Literature. Ann Glob Health, 2018. 84(4): p. 551-562.

45. Neufeld, L.M., et al., Global landscape of malnutrition in infants and young children, in Global Landscape of Nutrition Challenges in Infants and Children. 2020, Karger Publishers. p. 1-14.

46. De Onis, M., M. Blössner, and E. Borghi, Global prevalence and trends of overweight and obesity among preschool children. The American journal of clinical nutrition, 2010. 92(5): p. 1257-1264.

47. Ahmad, A., et al., Complementary feeding practices and nutritional status of children 6-23 months old: formative study in Aceh, Indonesia. Nutrition Research and Practice, 2018. 12(6): p. 512-520.

48. Egayanti, Y., N.S. Palupi, and E. Prangdimurti, Profile of complementary food consumption during the first year of life based on Indonesia Individual Food Consumption Survey 2014. Malays J Nutr, 2018. 24: p. 53-61. 
49. Dewey, K.G., Reducing stunting by improving maternal, infant and young child nutrition in regions such as South Asia: evidence, challenges and opportunities. Maternal \& Child Nutrition, 2016. 12: p. 27-38. 50. Moench-Pfanner, R. and M. Van Ameringen, The Global Alliance for Improved Nutrition (GAIN): a decade of partnerships to increase access to and affordability of nutritious foods for the poor. Food and nutrition bulletin, 2012. 33(4_suppl3): p. S373-S380. 